\title{
Exploration on Research Methods for Exploring Human Response to Luminous Environment with Chromogenic Windows Applied
}

\author{
Runqi Liang1, Yupeng $\mathrm{Wu}^{2}$, Jiawei Yao', Yongming Zhang3* \\ ${ }^{1}$ College of Architecture and Urban Planning, Tongji University, Shanghai 200092, China \\ 2Department of Architecture and Built Environment, University of Nottingham, Nottingham NG7 2RD, UK \\ ${ }^{3}$ Sino-German Institute of Engineering, Tongji University, Shanghai 200092, China \\ *Corresponding author: Yongming Zhang, zhangyongming@tongji.edu.cn
}

\begin{abstract}
Chromogenic windows enable both energy-saving and daylighting regulation. However, the human response to the luminous environment affected by them is difficult to test, since their features of dynamic change and tinting automatically. This study explores the research methods, including experimental design and statistical analysis, by literature review and an experiment demonstration. The results show that a proper size of the test room is significant to obtain desired data, and the advanced VR technologies have the potential to be applied for testing these dynamic variables. Bayesian approaches are recommended to be tried and get more accurate interference about the experimental results.
\end{abstract}

Keywords: Chromogenic windows; Building luminous environment; Human response; Statistical analysis; Experimental design

Publication date: May 2021; Online publication: May 31, 2021

\section{Introduction}

Over the last decades, sustainable building technologies have been well investigated. A large amount of energy use in buildings makes it sense that energy-saving approaches are desired. The chromogenic smart window (e.g., electrochromic, thermochromic, gasochromic, and photochromic) is a type of innovative approaches to regulating indoor thermal and luminous conditions ${ }^{[1]}$. That can reduce the undesired solar heat gain dynamically transmitted into the building, diminishing the possibility of overheating by solar radiation and achieving energy conservation simultaneously. Various studies have been undertaken to explore their energy performance, and electrochromic windows have commercial products, other types of chromogenic windows are still on the lab stage. Since the chromogenic windows have features of tinting with their solar transmittance changing during the work, such as blue, brown, etc., the indoor luminous environment is affected by the chromatic glazing as well. Human response to the daylighting conditions affected by the chromogenic windows is significant to be figured out before these innovative smart windows being applied in practice.

However, the features of 'dynamic' and 'tinting' improve the difficulties of experimental methods on human response to chromogenic windows, and statistical analysis influences the results as well. This study aims to explore the appropriate research methods specified for this type of dynamic chromatic glazing applied in working space, by using a self-designed experiment and literature review. It is proposed to give more particular suggestions to improve and accelerate the development of these energy-efficient smart windows. 


\section{Literature review of research methods}

This section demonstrated the research methods from three aspects, including experimental design for windows' application, statistical analysis tools, and innovative virtual reality (VR) technologies.

\subsection{Experimental design}

According to the size, the experiments for human response can be classified into two types:

(1) Small-scale, where participants can look into the test chamber and observe the given targets and complete questionnaires ${ }^{[2-3]}$.

(2) Full-scale, where participants are invited to walk into the test room and undertake some visual or non-visual tasks ${ }^{[4]}$.

Both methods are valid to obtain desired results, however, the subjective assessment about the whole indoor luminous environment by using a small-scale test room cannot restore the perception overall. the test of colour shifts affected by different window glazing (Figure 1. (a)) ${ }^{[5]}$, the observers are hard to receive all information indoors from the top view. While if a full-scale experiment was applied to test different types of windows, the change of window conditions will not be convenient as the small-scale one, as is shown in Figure 2. (b) ${ }^{[4]}$.

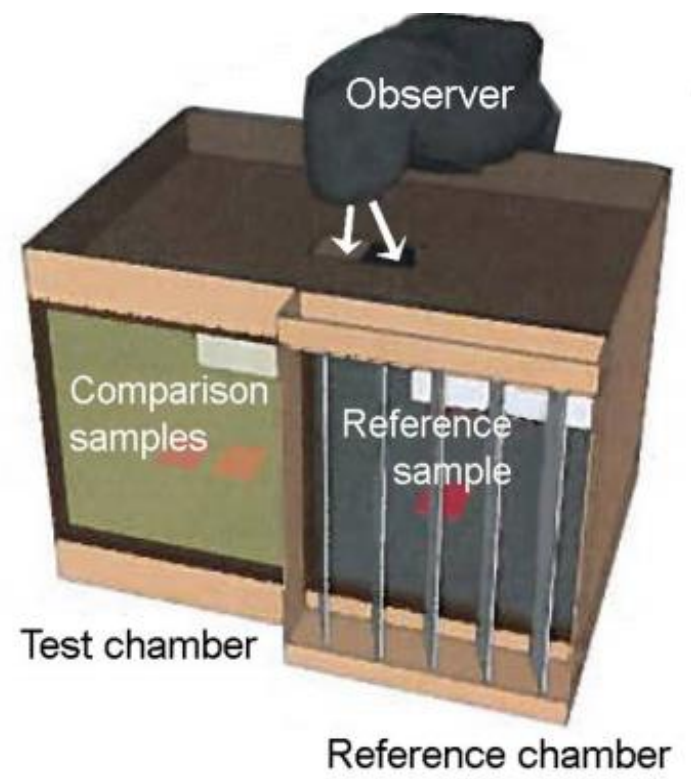

(a)
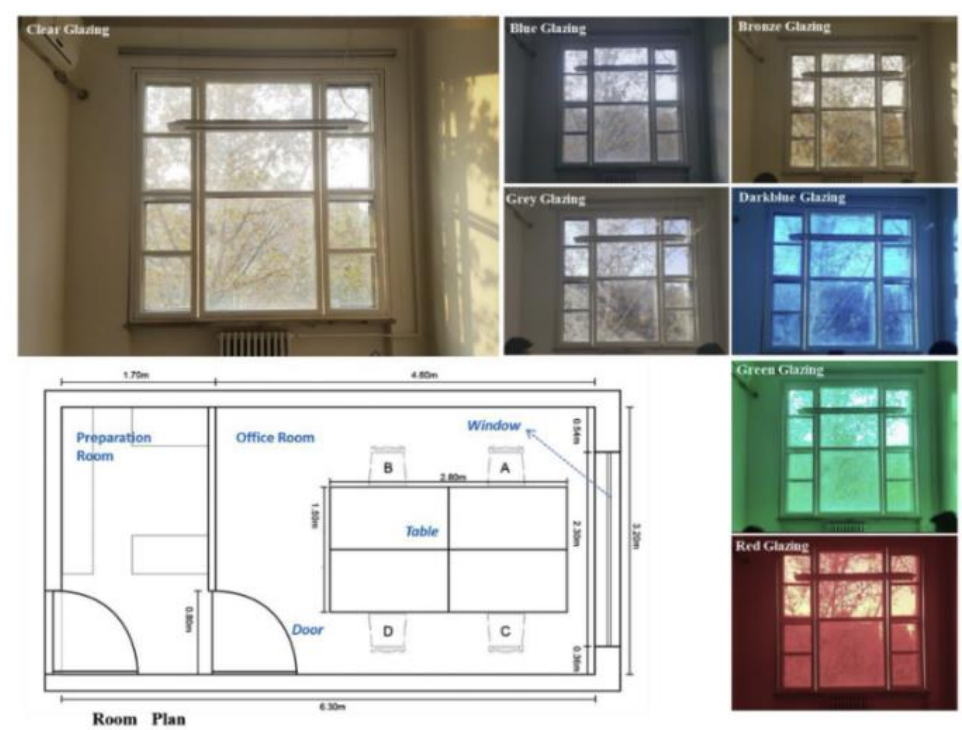

(b)

Figure 1. Two sizes of test room (a) small scale; (b) full scale ${ }^{[4,5]}$

\subsection{Statistical analysis}

Most of the evaluations on the luminous environment are based on the subjective response and objective performance from samples of occupants drawn from the building users. Therefore, statistical analysis has to be applied to deal with these results, in the perspective of analysis methods, most of the studies chose to use the classical frequentist approach, such as null hypothesis significance testing (NHST) depending on the sample size and magnitude of effect, to detect the results performance ${ }^{[6-8]}$.

Bayesian inference is proposed to be an alternative method to implement the analysis ${ }^{[9]}$. Different from frequentist approaches, Bayesian estimation can incorporate prior knowledge (i.e., based on existing knowledge), and combine the likelihood from the new data, resulting in a posterior distribution. In other words, Bayesian analysis is used to re-allocate the degree of belief across possibilities ${ }^{[9]}$. Additionally, the Bayesian hypothesis test can quantify evidence that the data provide for the null hypothesis (H0) and the alternative hypothesis (H1). Lee et al ${ }^{[10]}$ developed a Bayesian approach for providing accurate predictions 
for personalized thermal preference profiles. Another study conducted by Wang and Hong ${ }^{[11]}$ used the Bayesian inference approach to define new occupants comfort temperature range in office buildings of the USA. Xiong et al ${ }^{[12]}$ also use the Bayesian approach to infer the visual satisfaction profile in daylit office based on previously collected data from experiments. Therefore, it is believed that the Bayesian approach can be applied to validate experimental models and achieve more accurate inference based on known information.

\subsection{VR technologies}

Since the chromogenic windows have the features of dynamic changing of solar transmittance, which increase the difficulty of setting up and control the experiments. In the past decades, the combined use of advanced VR technology and building design have realized the test of user behaviour and achieve energysaving ${ }^{[13-14]}$. Shahinmoghadam et al. integrated the Internet of Things (IoT) and VR in the development of immersive VR applications for real-time monitoring of thermal comfort conditions. The prototype of the system has been implemented and used to support the consistency between the system output and the actual thermal sensation observed under various conditions ${ }^{[15]}$. Vittori et al. redesigned an office through VR and recorded the sense of presence, with a satisfaction rate of $76 \%$. The VR design environment is used to reproduce key variables of design, involving artificial lighting colour temperatures. VR evaluation significantly reduces the cost and achieves human-centred energy efficiency ${ }^{[16]}$. Yeom et al. used VR to determine the best window-to-wall ratio of buildings that can achieve energy efficiency and a healthy working environment ${ }^{[17]}$. It can be seen that VR is helpful for the building environment evaluation with multiple variables.

\section{An experiment of thermochromic windows}

The thermochromic (TC) window is one of the most promising smart windows that tints with temperature difference automatically, a series of experiments were designed to test the human response to the luminous environment with thermochromic window applied, exploring the specific experimental and analysis methods.

\subsection{Experiment setting up}

To achieve both Immersive experience of participants and convenient change of window conditions, a test cubicle with the dimension of $1.5 \mathrm{~m}$ (length)* $1.2 \mathrm{~m}$ (width)*2.1m (height) was built, and the indoor luminous affected by TC window were simulated by a virtual window so that the illuminance levels could be controlled manually. Three window conditions with two horizontal illuminance levels (100lux and 350lux):1) TC_Bronze, standing for $\mathrm{VO}_{2}$ nanoparticle film; 2) clear glazing as a reference; 3) TC_Blue, representing the iron-liquid based TC material, which can tint from clear to blue during the working. Detailed parameters of experimental conditions were shown in Table 1.

During the experiment I and II, 31 participants were invited to attend the test, respectively, they were required to complete objective tasks (Landolt rings visual test) and subjective assessment (questionnaires) under each window condition, the sequence of window conditions was randomized.

\subsection{Data analysis using Bayesian approaches}

The collected data were analysed by firstly using the classical frequentist approach for the experiment I and II separately, and the results show that TC_Bronze is relatively more acceptable in general. Since different participants were used for these two experiments, the both classical Mann-Whitney $U$ test and Bayesian approaches were tried to do the independent paired comparisons between I and II, to do exploration on analysis methods. For Bayesian approaches, BF10 present the evidence for supporting H0 (i.e., no 
difference) and H1 (i.e., difference significant).

Table 1. illuminance and colour temperatures (CCT) under three window conditions.

\begin{tabular}{lcccc}
\hline \multirow{2}{*}{ Window conditions } & \multicolumn{2}{c}{ Experiment I } & Experiment II \\
\cline { 2 - 4 } & Illuminance(lux) & CCT(K) & Illuminance(lux) & CCT(K) \\
\hline 1. TC_Bronze & & 4053 & & 4056 \\
2. Clear & 100lux $( \pm 5 \%)$ & 4903 & $3501 \mathrm{ux}( \pm 5 \%)$ & 4911 \\
3. TC_Blue & & 6934 & & 7054 \\
\hline
\end{tabular}

Table 2. Mann-Whitney U test and Bayesian Mann-Whitney U test of questions with significant difference between Experiment I and II.

\begin{tabular}{|c|c|c|c|c|c|c|c|c|}
\hline & \multirow{2}{*}{$\begin{array}{l}\text { Window } \\
\text { conditions }\end{array}$} & \multicolumn{2}{|c|}{ Mdn1-Mdn2 } & \multicolumn{3}{|c|}{ Mann-Whitney U test } & \multicolumn{2}{|c|}{ Bayesian one } \\
\hline & & 100lux & 350lux & Statistic & $\mathbf{p}$ & $\begin{array}{c}\text { Effect Size } \\
\left(\mathbf{r}_{\mathbf{B}}\right)^{\mathbf{a}}\end{array}$ & $\mathrm{BF}_{10}{ }^{\mathrm{b}}$ & $\mathbf{W}$ \\
\hline \multirow{3}{*}{ Brightness } & TC_Bronze & 2 & 3 & 271.5 & $0.002 *$ & -0.435 & $4.625^{*}$ & 689.5 \\
\hline & Clear & 3 & 4 & 266.0 & $0.002 *$ & -0.446 & $8.175^{*}$ & 695.0 \\
\hline & TC_Blue & 2 & 3 & 328.5 & $0.024 *$ & -0.316 & $1.682 *$ & 632.5 \\
\hline \multirow{2}{*}{ Uniformity } & TC_Bronze & 3 & 4 & 315.0 & $0.017 *$ & -0.344 & $2.869^{*}$ & 646.0 \\
\hline & TC_Blue & 2 & 4 & 335.0 & $0.035^{*}$ & -0.303 & $1.268^{*}$ & 626.0 \\
\hline \multirow{2}{*}{ Alertness } & TC_Bronze & 3 & 3 & 310.5 & $0.007 *$ & -0.354 & $1.658^{*}$ & 650.5 \\
\hline & TC_Blue & 3 & 3 & 330.0 & $0.027 *$ & -0.313 & $1.106^{*}$ & 631.0 \\
\hline \multirow{2}{*}{ Pleasantness } & TC_Bronze & 2 & 3 & 232.5 & $<.001 *$ & -0.516 & $8.982 *$ & 728.5 \\
\hline & TC_Blue & 2 & 3 & 328.0 & $0.023 *$ & -0.317 & $1.005^{*}$ & 633.0 \\
\hline Naturalness & TC_Bronze & 2 & 3 & 338.0 & $0.038^{*}$ & -0.297 & $1.285^{*}$ & 623.0 \\
\hline \multirow{2}{*}{ Comfort } & TC_Bronze & 2 & 4 & 237.5 & $<.001 *$ & -0.506 & $13.269 *$ & 723.5 \\
\hline & Clear & 2 & 3 & 288.5 & $0.004 *$ & -0.400 & $4.767 *$ & 672.5 \\
\hline \multirow{3}{*}{ Acceptance } & TC_Bronze & 2 & 4 & 244.0 & $<.001 *$ & -0.492 & $10.282^{*}$ & 717.0 \\
\hline & Clear & 2 & 3 & 262.5 & $0.001 *$ & -0.454 & $7.468^{*}$ & 698.5 \\
\hline & TC_Blue & 2 & 3 & 336.5 & $0.037 *$ & -0.300 & $1.549 *$ & 624.5 \\
\hline
\end{tabular}

* significant

a Effect size $\left(r_{B}\right): \quad r_{B}<0.3$ small effect, $0.3<r_{B}<0.5$ moderate effect, $r_{B}>0.5$ large effect.;

b Bayes Factor $\left(\mathrm{BF}_{10}\right) \quad$ : $0.33<\mathrm{BF}_{10}<1$ is anecdotal evidence for $\mathrm{H}_{0} ; \mathrm{BF}_{10}=1$ is no evidence; $1<\mathrm{BF}_{10}<3$ is anecdotal evidence for $\mathrm{H}_{1} ; 3<\mathrm{BF}_{10}<10$ is moderate evidence for $\mathrm{H}_{1} ; 10<\mathrm{BF}_{10}<30$ is strong evidence for $\mathrm{H}_{1} ; 30<\mathrm{BF}_{10}<100$ is very strong evidence for $\mathrm{H}_{1} ; \mathrm{BF}_{10}>100$ is decisive evidence for $\mathrm{H}_{1}$. 
It can be seen from the data in Table 2. that, brightness, uniformity, alertness, pleasantness, naturalness, comfort, and acceptance, the classical and Bayesian results are shown almost the same. However, classical results required both $\mathrm{p}$ and effect size $\left(\mathrm{r}_{\mathrm{B}}\right)$ to show the degree of the difference caused by illuminance levels, while $\mathrm{BF}_{10}$ shows the difference straightforward with the finer classifications. A 5-level Likert scale was applied to all answers to the questions, and 1 is for the most negative answer, and 5 for the most positive one. In terms of medians, especially for Brightness and Acceptance, participants gave more positive answers during experiment II than that of experiment I under all of the three window conditions. Moreover, the results show that the uniformity, alertness, pleasantness and comfort are all increased in experiment II with higher illuminance levels under the TC window conditions. That means that under the chromatic luminous environment, higher illuminance can lead to a more positive response. In Table 2., the cases with the effect size falling into the moderate range and $\mathrm{BF}_{10}$ falling into the anecdotal range indicate that the Bayesian method is more detailed to show the degree of illuminance effect.

\section{Conclusions}

This study explored the experimental methods and innovative analysis approaches by literature review and an experiment about the human response to the TC windows application, the experiment used a test cubicle, which is smaller than a full-scale room but it is accessible for participants. The proper size of the test cubicle enables the quick change of the window conditions as well. A review of VR technologies applied to optimize the building environment indicated that VR technologies have the potential to simulate daylighting conditions filtered by different window types, and creating various illuminance, CCT and other lighting conditions. Additionally, Bayesian approaches are suggested to be applied for getting more accurate inference, especially for these phased and batched experiments.

\section{Funding}

This work was supported by the project funded by the China Postdoctoral Science Foundation project (2019M661617), the Faculty of Engineering at the University of Nottingham through a PhD studentship awarded to Runqi Liang.

\section{Disclosure statement}

The author declares no conflict of interest.

\section{References}

[1] Jelle BP, Hynd A, Gustavsen A, Arasteh, et al., 2012, Fenestration of Today and Tomorrow: A StateOf-The-Art Review and Future Research Opportunities. Solar Energy Materials and Solar Cells. 96: $1-28$.

[2] Vossen FM, Aarts MPJ, Debije MG, 2016, Visual Performance of Red Luminescent Solar Concentrating Windows in an Office Environment. Energy and Buildings. 113: 123-132.

[3] Dubois MC, Cantin F, Johnsen K, 2007, The Effect of Coated Glazing on Visual Perception: A Pilot Study Using Scale Models. Lighting Research and Technology. 39(3): 283-304.

[4] Chen X, Zhang X, Du J, 2019, Glazing Type (Colour and transmittance), Daylighting, and Human Performances at a Workspace: A Full-Scale Experiment in Beijing. Building and Environment. 153: 168-185.

[5] Matusiak B, Anter KF, Angelo K, 2012, Colour Shifts Behind Modern Glazing. University College 
of Arts, Crafts and Design, Stockholm, available online: https://www.researchgate.net/publication/263470151_Colour_shifts_behind_modern_glazing

[6] Yu H, Akita T, 2019, The Effect of Illuminance and Correlated Colour Temperature on Perceived Comfort According to Reading Behaviour in a Capsule Hotel. Building and Environment. 148: 384393.

[7] Ru T, de Kort YAW, Smolders KCHJ, et al., 2019, Non-Image Forming Effects of Illuminance and Correlated Colour Temperature of Office Light on Alertness, Mood, and Performance Across Cognitive Domains. Building and Environment. 149: 253-263.

[8] Revantino, Mangkuto, et al., 2018, The Effect of Illuminance and Correlated Colour Temperature, and Colour Rendering of Various Existing Light-Emitting Diode Lamps on Subjective Preference and Performance in Indonesia. Journal of Building Engineering. 19: 334-341.

[9] Kruschke JK, Liddell TM, 2017, The Bayesian New Statistics: Hypothesis Testing, Estimation, Meta-Analysis, and Power Analysis from a Bayesian Perspective. Psychonomic Bulletin \& Review. 25(1): 178-206.

[10] Lee S, et al., 2018, A Bayesian Approach for Probabilistic Classification and Inference of Occupant Thermal Preferences in Office Buildings. Building and Environment. 118: 323-343.

[11] Wang Z, Hong T, 2020, Learning occupants' indoor comfort temperature through a Bayesian inference approach for office buildings in United States. Renewable and Sustainable Energy Reviews. 119: 109593.

[12] Xiong J, et al., 2018, Inferring Personalized Visual Satisfaction Profiles in Daylit Offices from Comparative Preferences using a Bayesian Approach. Building and Environment. 138: 74-88.

[13] Abd-Alhamid F, et al., 2020, Evaluating the Impact of Viewing Location on View Perception using a Virtual Environment. Building and Environment. 180.

[14] Flor JF, et al., 2021, Virtual Reality as a Tool for Evaluating User Acceptance of View Clarity Through ETFE Double-Skin Façades. Energy and Buildings. 231.

[15] Shahinmoghadam M, et al., 2021, BIM- and IoT-Based Virtual Reality Tool For Real-Time Thermal Comfort Assessment in Building Enclosures. Building and Environment. 199.

[16] Vittori F, et al., 2021, Subjective Thermal Response Driving Indoor Comfort Perception: A Novel Experimental Analysis Coupling Building Information Modelling and Virtual Reality. Journal of Building Engineering. 41.

[17] Yeom S, et al., 2020, Determining the Optimal Window Size of Office Buildings Considering the Workers' Task Performance and the Building's Energy Consumption. Building and Environment. 177. 